\title{
New approach to detect imminent slope failure by utilising coherence attribute measurement on ground-based slope radar
}

\author{
FA Cahyo GroundProbe, Indonesia \\ R Dwitya GroundProbe, Indonesia \\ RH Musa GroundProbe, Indonesia
}

\begin{abstract}
Detecting imminent failure is immensely important in slope monitoring, especially in open pit mining where the hazard potential can severely affect safety and production. The current, well-accepted method to detect an imminent failure is to monitor the slope behaviour in the wall and a prediction can be made from the interpretation of progressive slope deformation behaviour prior to collapse. This paper will convey a new approach that can be developed to detect an imminent failure with precise results by utilising the coherence attribute produced by the ground-based slope radar. Coherence in ground-based slope radar implies the normalised complex cross-correlation function of amplitude and range measurements. The coherence measurement can be utilised to determine how much the wall surface on each pixel has changed between successive radar scans. Development of slope deformation from a progressive trend into failure event will yield increasing percentage of low coherence pixel over time.
\end{abstract}

This paper summarises the result of the back-analysis of three slope failures, where the coherence value of each pixel on the area that had undergone failure is retrieved and arranged into three coherence threshold values of 0.990, 0.995, and 0.999. Then each group is summed to know the number of pixels and subsequently, the percentage of total failure areas which have coherence values shifting below those three coherence thresholds over the time of radar scans. A thorough analysis toward the shifting of low coherence pixels percentage over time, which generally shows a progressive trend on the chart, could detect the imminent failure and provide the notification prior to the real failure with varied time-lapse from 0.8 hours to more than 12 hours based on the selected study cases. Certain interpretation towards this coherence method, by taking into account its limitations as well, helps to ensure detection of imminent failure as well as complement other common failure detection methods that would otherwise be more complex and uncertain without it.

Keywords: failure, slope stability radar, coherence, range, amplitude, low coherence pixels, alarm threshold

\section{Introduction}

Ground-based slope radar utilises interferometry in order to measure deformation of slopes or other objects of interest. Detecting imminent failure is immensely important in slope monitoring, especially in open pit mining where hazard potential from it could severely affect safety and production. Open pit mine failure, which contributes a significant portion of accidents leading into both fatality and substantial financial loss, is a major factor that needs to be well-anticipated. A particular technology needs to be applied in order to detect the cluster of the area in which a failure potential may arise. Moreover, the detection could be further developed into failure prediction that could assist the efforts for mitigation. At any rate, detecting an impending failure is the first and the most crucial step in slope stability monitoring, as this gives time for evacuation and other mitigation efforts to address the failure. 
This paper will discuss a new method in detecting an impending failure based on one of ground-based slope radar attribute measurements called coherence, as both an alternative and a supplement to the more distinguished methods to detect failure. Acceleration on slope deformation is the preamble sign prior to failure. This is known as progressive deformation behaviour. If someone working with slope stability monitoring detects this kind of slope deformation behaviour-whether this detection is inferred from radar or other tools for monitoring-this can be perceived as a legitimate sign that an impending failure is about to happen. There is consensus among slope stability analysis practitioners that that impending failure can be detected by observing progressive deformation behaviour. Coherence measurement from ground-based slope radar, based on the experience and prior research that forms the foundation of this paper, could play a similar role as progressive deformation behaviour in detecting an impending failure. How coherence could provide such information about an impending failure along its limitation, but also its advantages compared to detecting impending failure using progressive slope deformation behaviour, will be discussed thoroughly in the paper.

\section{Radar interferometry for slope monitoring}

Interferometry is defined as a method to extract information from interference phenomenon of electromagnetic waves. This method found its path to assist deformation monitoring during early of 1980 in which an interferometric synthetic aperture radar (InSAR) is utilised to monitor vertical \& horizontal motion of Rutford Ice Stream of Antarctic (Goldstein et al. 1993). The method that latter recognised as InSAR, utilise phase comparison of radar signals attained from a pair of (synthetic aperture radar) SAR images taken a few days apart to plot an interferogram (Goldstein et al. 1993). The result of comparison apparently implies that phase difference between successive radar signals could be further calculated to measure deformation or displacement. The application of SRI in this light revealed that half a wavelength of displacement $\left(2.8 \mathrm{~cm}\right.$ on that case) generated a phase of $360^{\circ}$. Hence the phase difference recorded will be pertinent with the amount of displacement over the time span of two radar signals being compared. The success of InSAR to detect minute movement inspired the incorporation of interferometry method on the ground-based slope radar (Noon et al. 2001).

Ground-based slope radar is devised by combining the concept of measuring minute deformation by interferometry method along with the flexibility nature of its deployment in order to suit the requirement for slope stability monitoring especially in mining industry. Instead of using SAR hovering at certain location above the observed object (as per example of InSAR on paragraph above) or place it at a moving platform, the ground-based slope radar utilises real aperture on a stationary platform. The ground-based slope radar discussed in this paper will produce a narrow width beam (around $2^{\circ}$ ) and $31.4 \mathrm{~mm}$ of wavelength. This radar conducts a raster scan, in which the radar antenna will rotate in two dimensions (azimuth and elevation). The radar system will then dissect the whole scan area into cluster of independent pixel (Noon et al 2001). The size of the pixel will depend on the range from the radar antenna to the area observed. Henceforth comparison of phase angle of each pixel from successive scan will be done, that generate the magnitude of displacement exemplifies by the wall. The mechanism of the interferometry and the attribute of the wavelength are as such that the maximum deformation that could be measured correctly by the radar is $7.85 \mathrm{~mm}$. Displacement that exceeding this value, will lead into ambiguity or the lost track of the real magnitude of the displacement.

Interpretation of the deformation behaviour on ground-based slope radar deformation will give subtle information for slope stability monitoring purposes. Vast amounts of observation and research throughout the world in regards to nature of mining wall rock mechanic lead to one conclusion. Small precursor movement of the rock wall will occur on varied extended periods (depending on many factors such as the lithology, geological structure, external force, etc.) before the rock mass failure take place. Furthermore, observation toward the deformation behaviour preceding the failure event could give insight about detection and prediction of failure in the future. There are three types of slope deformation behaviour delineated on the pretext of the velocity signature that it possessed (Zavodni \& Broadbent 1980). Linear deformation behaviour occurs when the wall exhibit an outward movement with constant velocity, 
regressive deformation behaviour occurs when the wall exhibit an outward movement with decreasing velocity at fixed time span, and progressive deformation behaviour occurs when the wall exemplifies an outward movement with increasing velocity over a particular time span. These behaviours can be clearly observed through the eye of the radar. Typical tell-tale signs of a failure encompasses linear deformation behaviour followed by progressive deformation behaviour that will finally lead into a failure event. Simply put, progressive deformation behaviour is the premonition of the failure that about to come, or in other words, one can detect the possibility of impending failure from it. Furthermore an inverse velocity value inferred from the rate of the slope movement could be utilised in such way to predict the time of failure on some extent (Fukuzono 1985). In the next part of the paper, a new method to detect impending failure utilising coherence measurement will be explain.

\section{Coherence measurement on ground-based slope radar}

Coherence measurement in ground-based slope radar is inherently related with both amplitude and range value. Thus, to have a better understanding about this on the next paragraph amplitude and range concept on ground-based slope radar will be discussed.

\subsection{Amplitude}

Amplitude can be regarded as the height of the wave which will determine the intensity of the wave energy. Observing the figure of wave geometry in Figure 1, it can be inferred that amplitude is the measurement of the top half of a wave. The amplitude of a light wave implies a measure of how much energy the wave carries. Ground-based slope radar constantly measures the amplitude of the reflected wave signal, as the result when the transmitted wave signal is colliding with a solid object the radar observed. Amplitude measurement by the ground-based slope radar will signify the strength of the returned signal received by it that will give a cue about the object currently being observed by the radar. High magnitude of amplitude measurement will signify a solid object not uncommonly metal, while the low magnitude amplitude measurement will signify object located at far range or perhaps the wave signal travel into open sky. The magnitude of a particular slope wall-which obviously becomes the object of concern for the ground-based slope radar-lies between the former and the latter. Ground-based slope radar amplitude measurement is based on the magnitude of the resultant of the phase vector formed by the incoming signal.

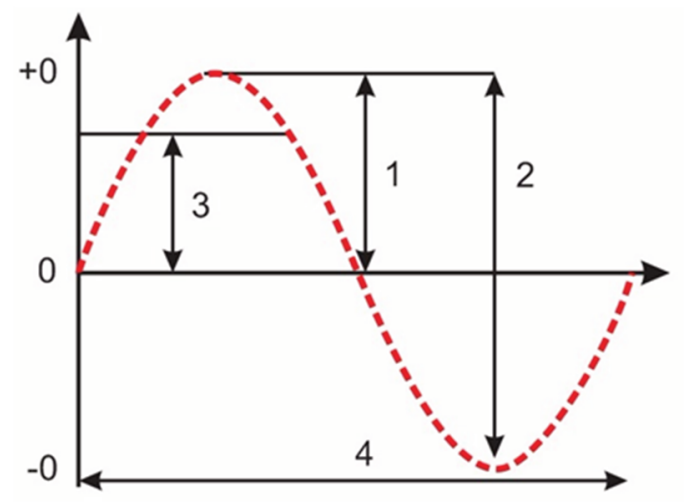

Figure 1 Geometry of sinusoidal wave: (1) Amplitude (2) Peak to peak amplitude; (3) Root mean square amplitude; (4) Wave's period

\subsection{Range}

Range is typical radar terminology which holds particular meaning related to the distance between the radar and the observed object (Skolnik 2001). Range in ground-based slope radar is therefore defined as the distance measured from the radar towards the observed target, based on wave signal time-of-flight, to the certain area or point within the pixel which has the stronger energy return. In other words, which has the highest amplitude. Hence, the range to a targeted pixel is determined by the time it takes for the 
ground-based slope radar signal to travel to the target and back. The speed of light, which is $\mathrm{c}=3 \times 10^{8} \mathrm{~m} / \mathrm{s}$, is the velocity of electromagnetic energy such as radar wave signal to travel in free space. If the time for the signal to travel to a target located at a range $R$ and return back to the radar is $2 R / c$, the range of the targeted radar pixel is then:

$$
R=\frac{c T}{2}
$$

where:

$$
\begin{aligned}
& \mathrm{R}=\text { range. } \\
& \mathrm{C}=\text { speed of light }\left(3 \times 10^{8} \mathrm{~m} / \mathrm{s}\right) . \\
& \mathrm{T}=\text { time required for radar signal to be transmitted and reflected back to radar aperture. }
\end{aligned}
$$

\subsection{Coherence}

Coherence value on the ground-based slope radar reveals the consistency of amplitude and range. It will have a value from the range of 0 to 1 . Coherence value equal to 1 means that the surface of the area monitored by the ground-based slope radar is intact and undisturbed. Any surface disturbance at the area being monitored by ground-based slope radar will be expressed by drop in coherence value below 1 . For example, based on experience, if there is a droplet of water on the slope surface, ground-based radar will show coherence value around 0.95 towards that area. Rockfall will usually generate coherence measurement around 0.8, while the major slope failure event will further alleviate coherence value into below 0.2. Therefore, coherence will show the consistency of the surface being monitored by ground-based slope radar from one scan to the next scan. The system makes it possible to measure coherence, by creating a graph show on Figure 2 below. The system will plot amplitude on $Y$-axis and range on $X$-axis. Thereafter, the program inside the ground-based slope radar will scrutinise the pattern of this amplitude range graph from one scan to its successive scan.

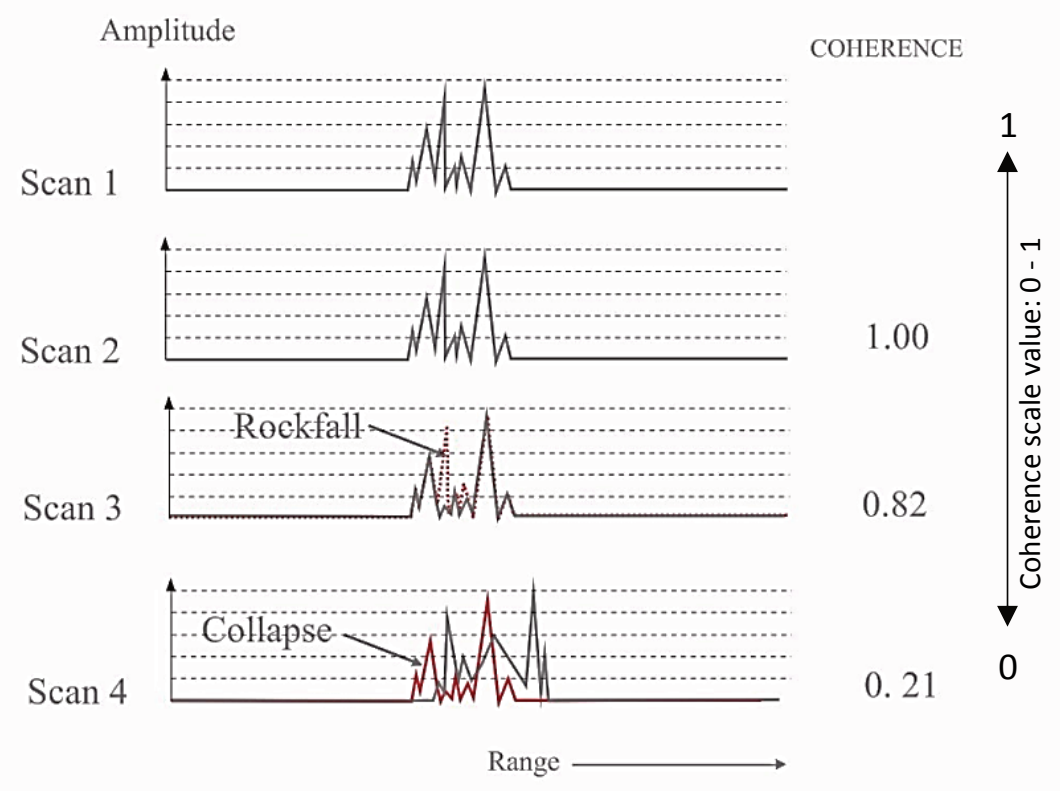

Figure 2 Simplified concept of coherence measurement in ground-based slope radar

In order to generate coherence value of each scan, ground-based slope radar system will conduct a normalised complex cross-correlation function of amplitude signature in its relation with range measurement. To make it easy to understand this concept, it can be envisaged that ground-based slope radar will compare the amplitude signature as function of range from one scan to another successive scan. If the amplitude signature and range measurement of one scan almost perfectly resembles the next scan, 
ground-based slope radar will bestow the maximum value of coherence which is 1 . Moreover, if the comparison between amplitude signatures and the distance of the wall or object from ground-based slope radar in a successive scan exhibits a degree of difference, the ground-based slope radar system will yield a low coherence value. The scale of coherence value is from maximum 1 to minimum 0 . The more haphazard the difference of the amplitude signature between one scan to the next scan is, then the lower the coherence value will be. It can be understood that a shift in coherence will tell whether a surface of a slope, or in more detail surface in one radar pixel, is changing or not. A stable value which is close to maximum of coherence over particular time span implies that the surface contained in particular pixel didn't changed. An erratic shift in coherence value measurement could mean that the surface of particular pixel is changing. Elaborating the concept of coherence measurement by ground-based slope radar could lead into impending failure detection as will be discussed later.

\section{$4 \quad$ Detecting impending failure using coherence from three selected cases}

In order to elaborate how coherence value measurement by ground-based slope radar could best serve the purpose of failure detection, it is important to emphasise the nature of coherence value shifting when a wall is approaching failure event. Despite the previous mention that a particular range value of coherence could signify the condition of wall being observed, detecting failure by observing the drops in coherence value of each pixel will prove to be unfruitful. This is based on the fact that a significant drop in coherence value is not happening in a progressive fashion, yet it happens in a relatively quick instance. As mentioned, failure events will commonly coincide with a drop of coherence value from a high coherence value, above 0.95, into the range of below 0.2 . This deflation can happen averagely only after 2-3 radar scan times. Depending on the distance between radar and the observed wall, this can be equal to 10-15 minutes only. Hence, a radar alarm which invokes warning if particular pixels are experiencing a drop in coherence value with predetermined parameter will provide insufficient lapse time for any effort to mitigate the impending failure. Henceforth, rather than assigning a certain parameter based on the deflation of coherence value, the method that will be conveyed in this paper will benefit from the sensitivity of coherence reading conducted by ground-based slope radar.

Rigorous observation towards coherence image shifting signature from selected study cases during the sequence of progressive deformation behaviour leading into failure signifies that there is a gradient of progression. On the ground-based slope radar system, to ease the observation of coherence value shifting, an interface of coherence image is created. This coherence image will depict the coherence value of each on single pixel as the representation of colour gradient depends on the threshold parameter being set. The default gradient is a high coherence value and will be represented by a lighter colour while the opposite dark colour is the representation of a low coherence value. The method of detecting impending failure using coherence is best explained by this feature of coherence image interface in ground-based slope radar system. The tenet that becomes the cornerstone of this paper is that during the sequence of slope wall deformation, as witnessed and observed by the ground-based slope radar which in turn will develop into a failure, will clearly expressed at the coherence image as shifting from lighter into darker tone of coherence image and it is reasonably detectable. In this sense, the word detectable emphasises that the coherence image shift is not of a sudden nature and therefore, observing and assigning some parameters to trigger an alarm on the coherence image shifting will provide an adequate time span for any failure mitigation effort. Strictly speaking, one can detect an impending failure visibly by observing this coherence shifting phenomenon. The method to detect the imminent failure by utilising this phenomenon will be coined as 'coherence shifting method'.

\subsection{Study case 1}

Study case 1 exhibits a sequence of failure taking place at a coal mining setup where the slope wall is a soft rock type designation based on the framework of rock mechanic. The progressive deformation behaviour has been clearly emerging from the start of the ground-based slope radar scanning period. The delineation 
of pixels which exhibits this progressive behaviour resulted in a cluster consisting of 120 pixels. The coherence value of all the selected pixels is retrieved, which will reveal how the coherence value of each pixel transpired over the time of the radar scan. At each radar scan interval, the pixels which depict coherence value deflation under $0.999,0.995$, and 0.990 (the three selected threshold value based on trials) will be counted and converted into percentage value. Therefore, one can mathematically observe the nature of coherence value shifting during the slope wall sequence of deformation leading into the impending failure. The graphic projected from the percentage of pixels which experience this coherence value deflation under the determined threshold will be called 'coherence shifting graphic' for simplicity. This case shows a clear gradation of coherence image shifting from quite early in the radar scan. The changing tone of the coherence image from light to dark happened quite haphazardly, presumably due to the fact that the mechanism of the failure belongs to the creep failure mechanism (Figure 3). Taking a look at the coherence shifting graphic, several rain events also inflicted a deflation of coherence value that is of a sudden nature (Figure 4). However, approaching the ultimate failure, the shifting progression became more obvious, especially for around the eight hours prior to the failure.

\section{Radar Coherence Image}

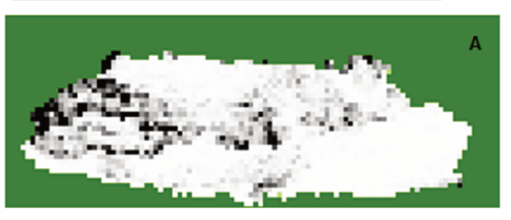

Monday, 21 November 2016

逍 $07: 02: 47$ -

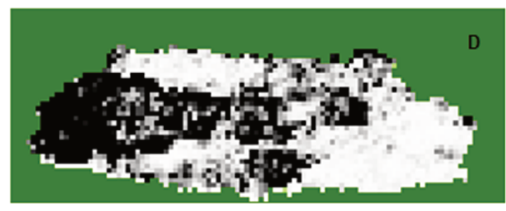

Thursday, 24 November 2016 进 08:10:17

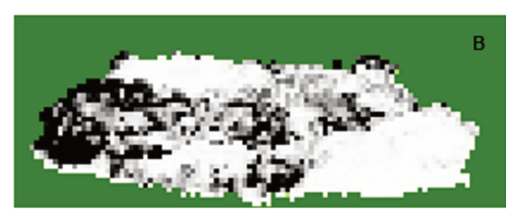

Wednesday, 23 November 2016 분 06:27:14 -

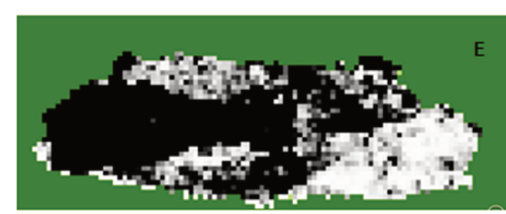

Thursday, 24 November 2016 通 18:31:53 -

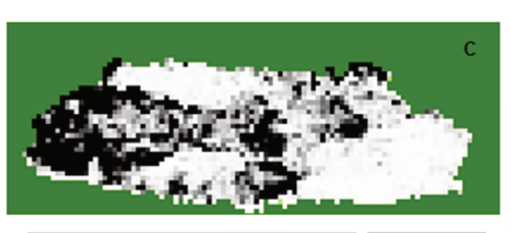

Wednesday, 23 November 2016 逍 21:58:58

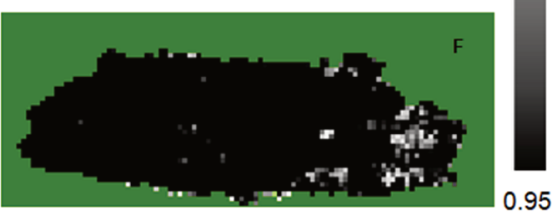

Thursday, 24 November 2016 这 20:46:02 -

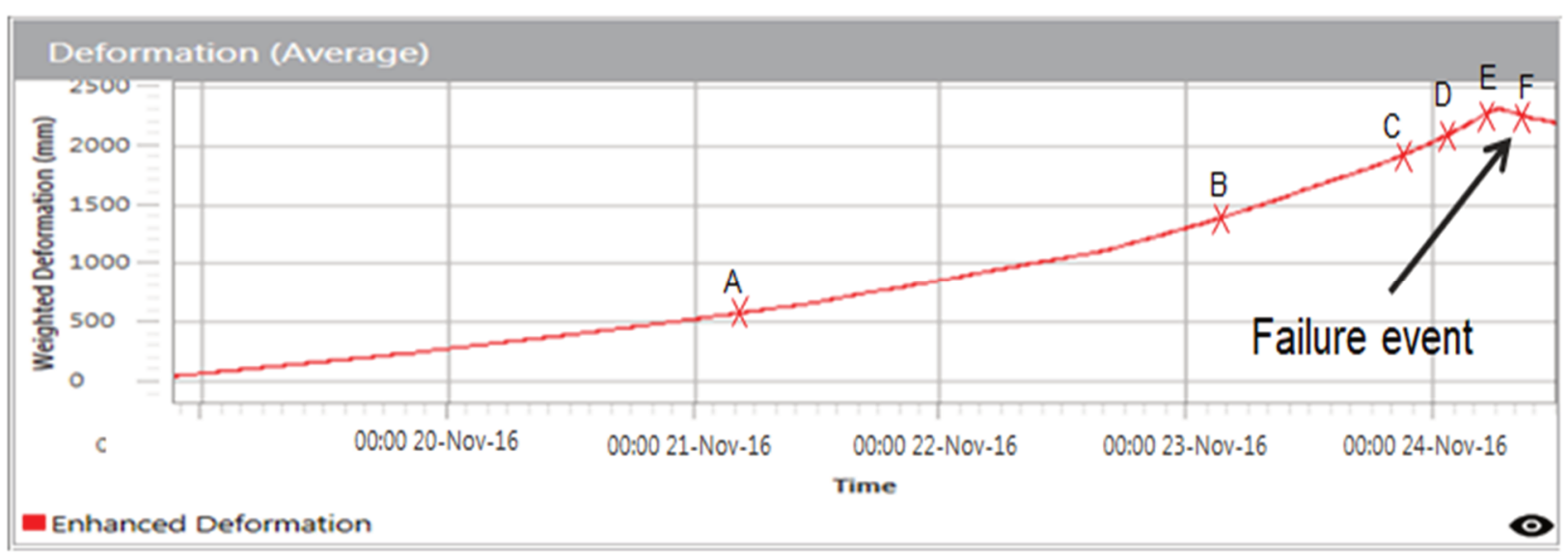

Figure 3 Coherence shifting image from ground-based slope radar study case 1 


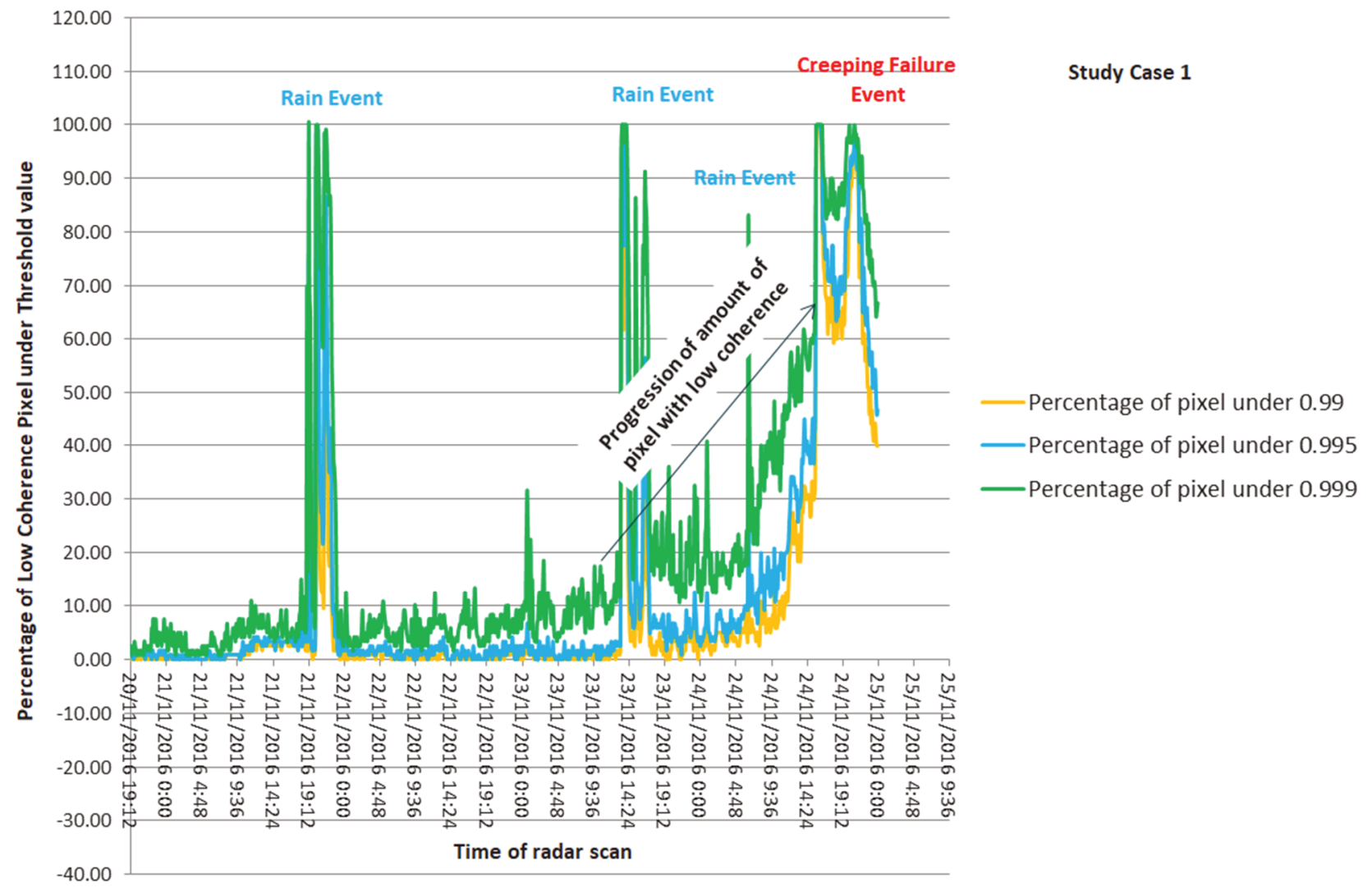

Figure 4 Coherence shifting graphic from ground-based slope radar study case 1

\subsection{Study case 2}

Study case 2 depicts an example of creeping failure in which the rate of deformation is too fast for the ground-based slope radar to track. Despite this fact, the failure event in this case is still easily detected by coherence in which the gradation of light coherence image progressively into darker coherence image is quite subtle over time. Almost every pixel contained inside the area of failure shows a progressive deformation behaviour which turns into ambiguity several hours prior to the failure event. If one merely utilises the progressive deformation to detect the failure, an incorrect conclusion can be inferred as to when the failure will occur. As the ambiguity happens, the chart of deformation from ground-based slope radar is no longer reliable since the radar has already lost track of wall deformation. An inept interpretation could suggest that the failure already occurred when this ambiguity occurred because it is not uncommon for a failure event to coincide with the progressive deformation curve tip off to ambiguity. If the interpretation involves coherence analysis, one might know that the deformation process is actually still developing, even after the ambiguity. The failure thereafter eventually occurred on 8 December 2017 at 9:29 am, where all the pixels show a dark coherence image. The shifting of the coherence image showed that some part of the outer rim of the failure area experienced the deflation of coherence earlier than the centre part (Figure 5). This presumably implies that failure in this case is controlled to some extent by geological structures. The aforementioned outer rim is possibly the sheared zone or cracks which trigger this failure. The coherence shifting graphic from this case depicts visible progression from high coherence to low coherence (Figure 6). 


\section{Radar Coherence Image}
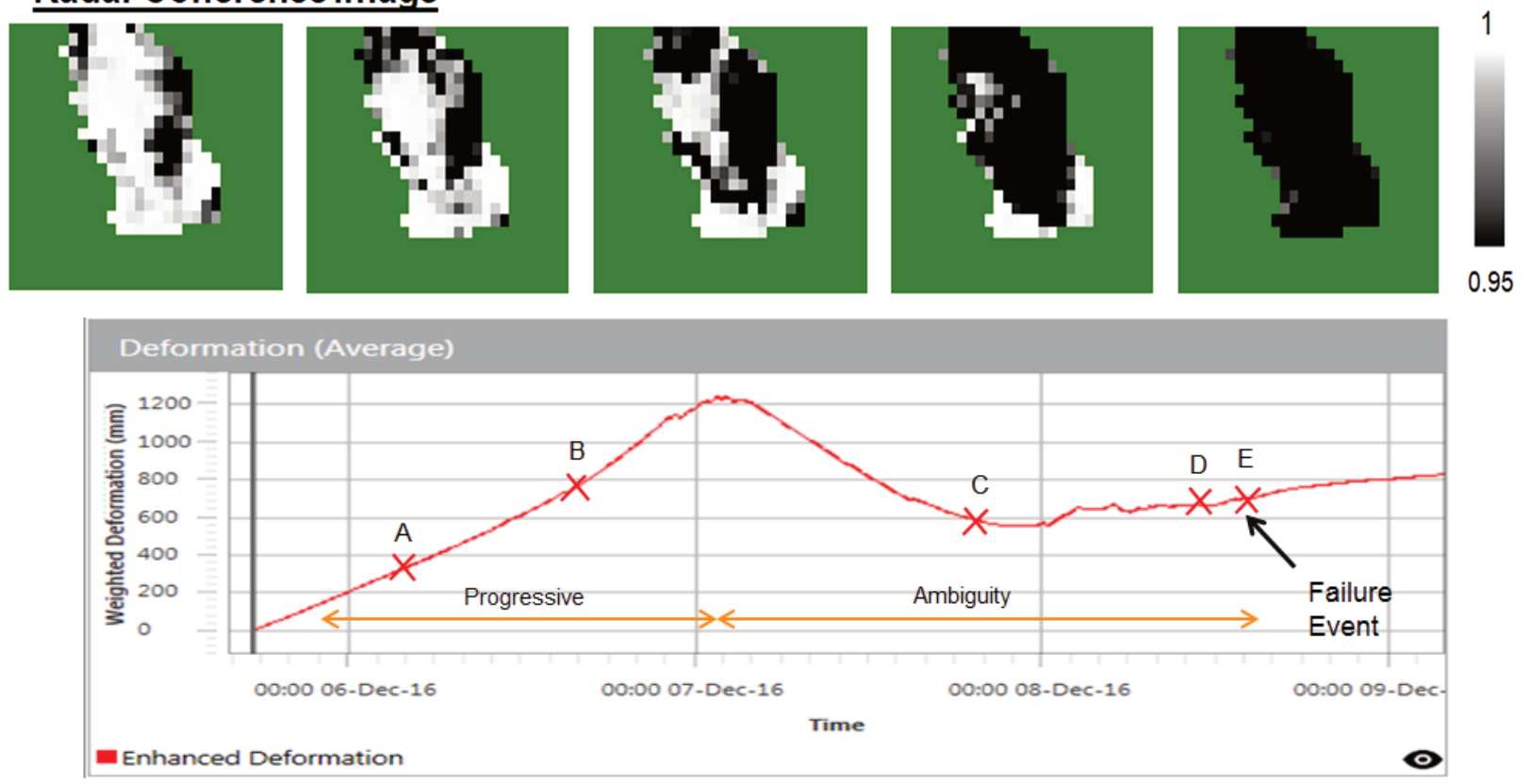

Figure 5 Coherence shifting graphic from ground-based slope radar study case 2

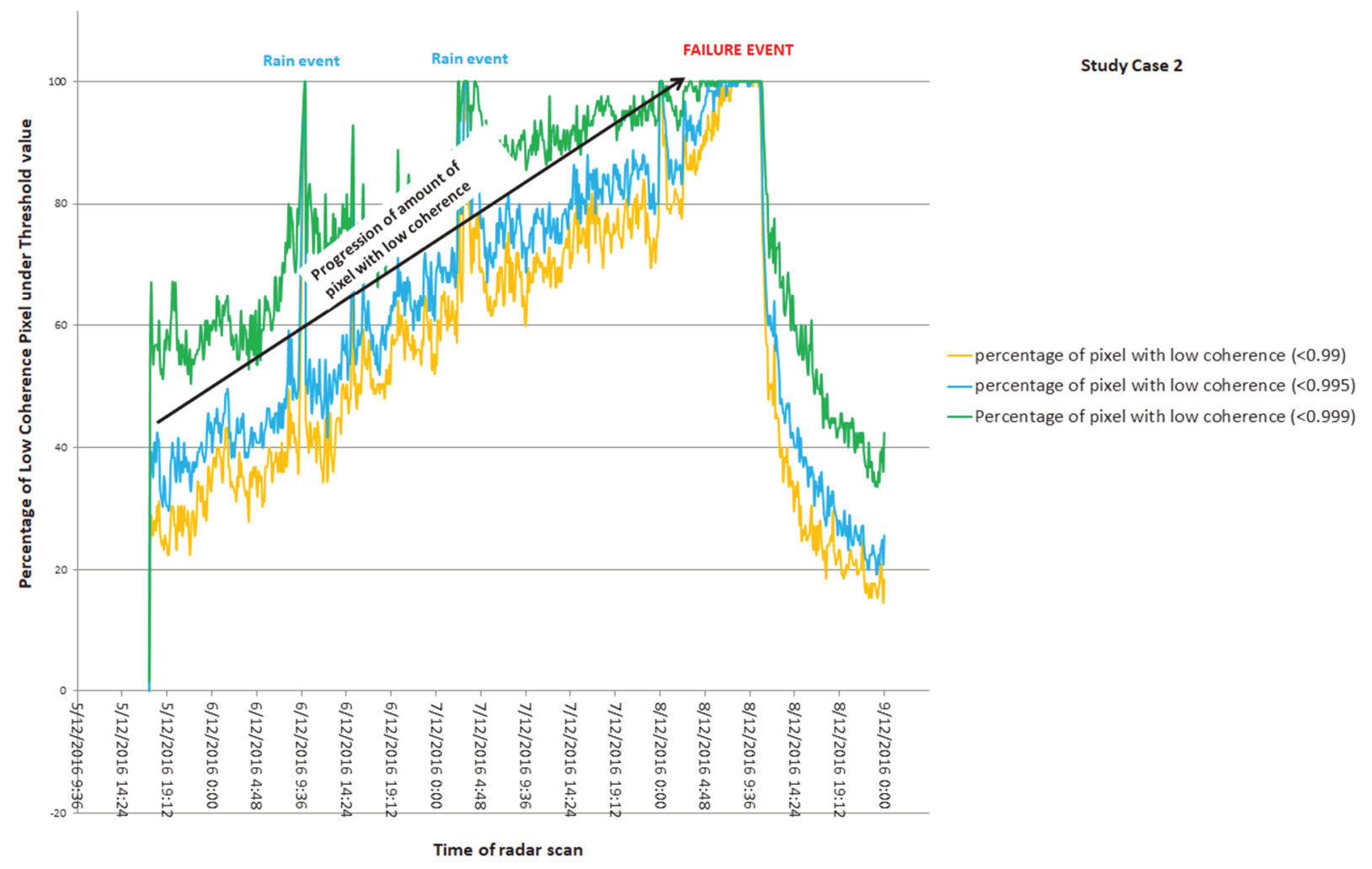

Figure 6 Coherence shifting graphic from ground-based slope radar study case 2

\subsection{Study case 3}

Study case 3 takes place at a mining area with a typical hard rock system which will affect how the coherence shifting will develop. The time lapse from the initial failure potential detection until the failure event by coherence shifting method only yielded a relatively short time span compared with other cases in this paper. Notwithstanding this fact, this case still provides insightful information regarding failure detection. Hard rock system, as usually encountered in gold and diamond mines, inherently exhibits failure 
which is typically preceded by short-term progressive deformation, also compared to the soft rock system. Therefore, it is not odd for coherence shifting method to yield a failure potential detection with such nature as well. The way the coherence image shifts from light to dark shade is inwardly thrusting from the outer rim (Figure 7). This phenomenon signifies that the failure is under the influence of a geological structure. Based from the photo observation, the failure is of wedge type. The coherence shifting graphic exemplified a progression curve of the pixel percentage which possessed a coherence value under the threshold as the radar scan time progressing (Figure 8). The curve representing coherence threshold value of 0.999 detected the failure potential at its best, compared with other curves as it starts to progress earlier.

\section{Radar Coherence Image}

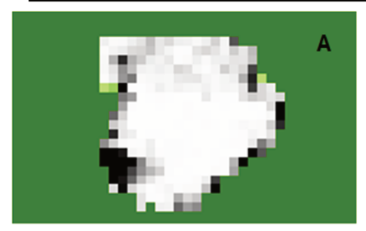

Sunday, 24 August 2008 苞 23:51:05 -

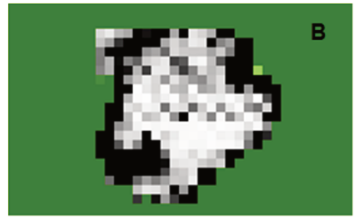

Monday, 25 August 2008 圈 01:08:26 -

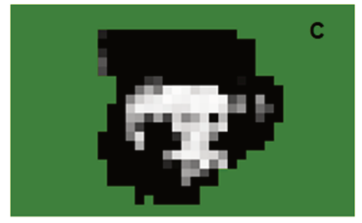

Monday, 25 August 2008 圈 01:56:08

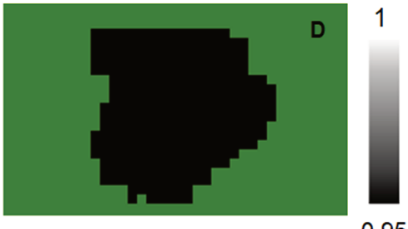

Monday, 25 August 2008 国 02:19:54 一

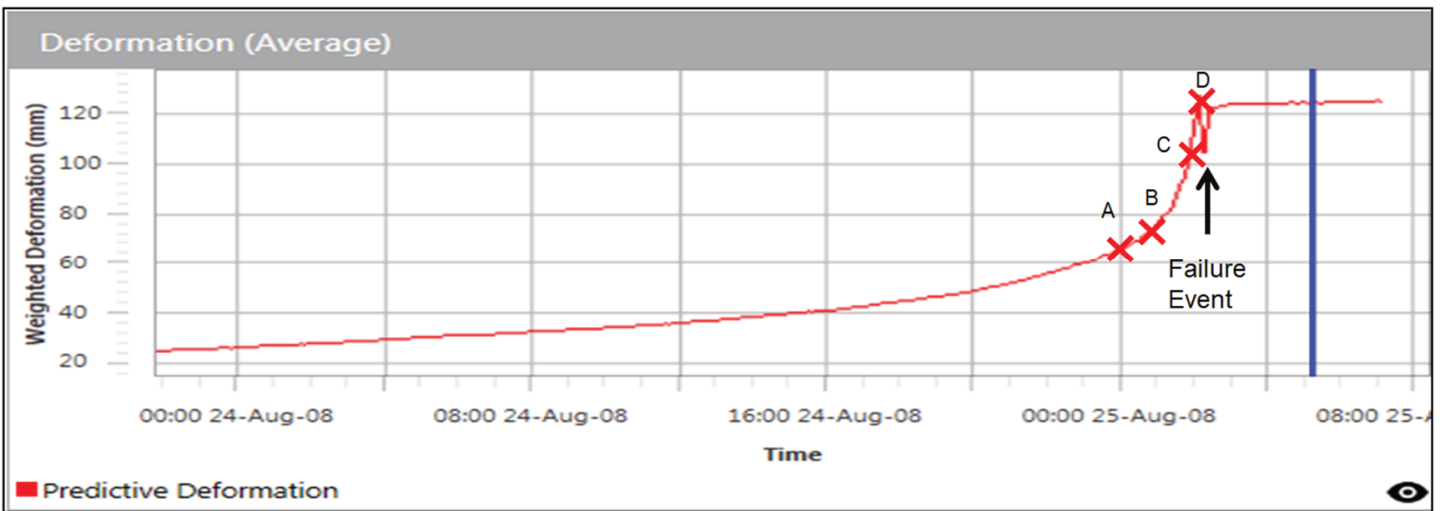

Figure 7 Coherence shifting graphic from ground-based slope radar study case 3

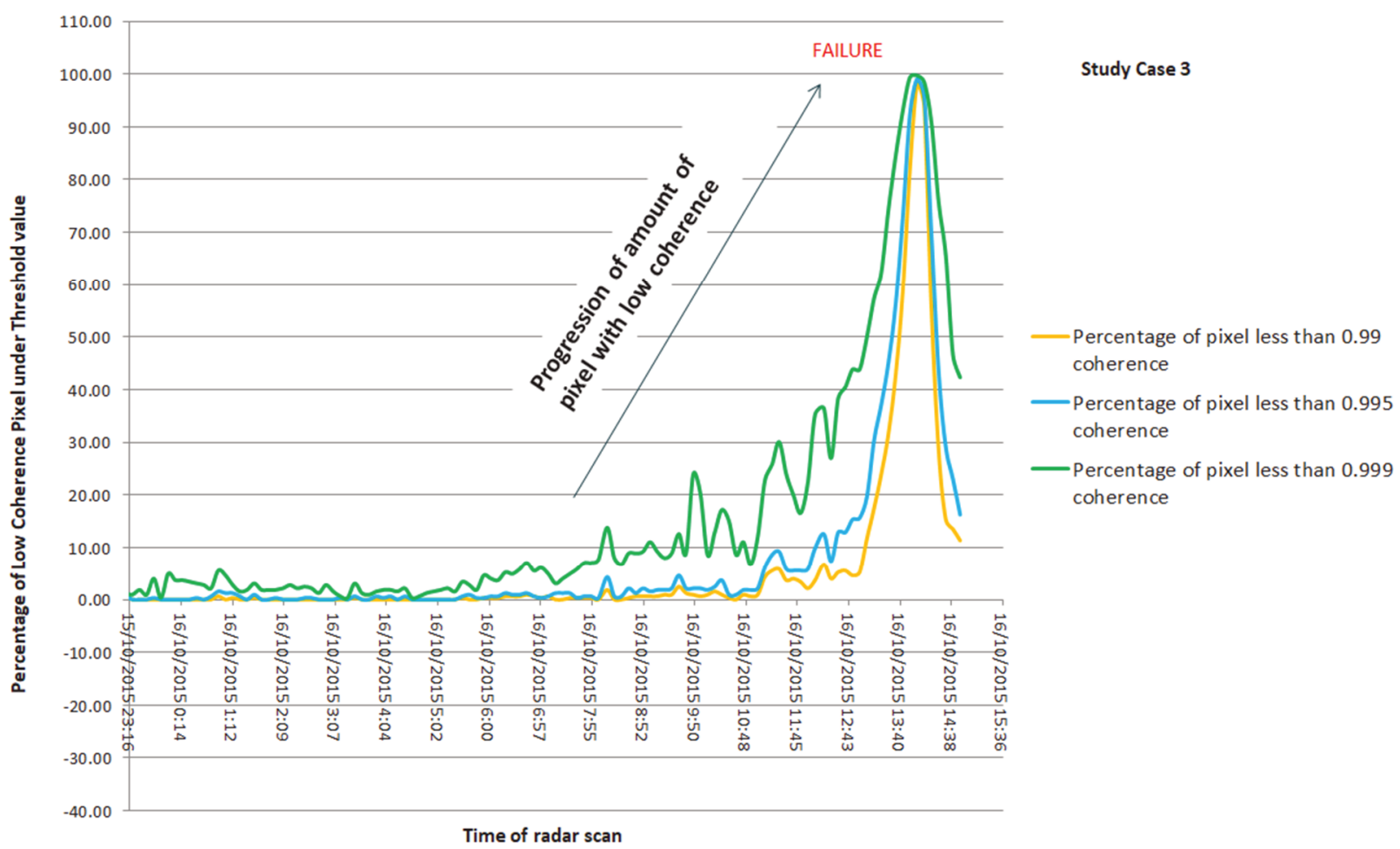

Figure 8 Coherence shifting graphic from ground-based slope radar study case 3 


\section{$5 \quad$ Alarm setup using coherence shifting method}

To enhance the ability to detect the impending failure utilising this coherence shifting method, an alarm system is applied with several settings. There are three parameters which need to be emphasised. These are the coherence threshold parameter $(0.999,0.995$, and 0.990$)$, the amount of pixel experiencing coherence deflation under predetermined threshold (depending on the total pixel of failure area, a percentage of it will be applied and converted: for soft rock system it will be 25,50 , and $75 \%$, while for hard rock system it will be 15,25 , and $50 \%$ ), and number of radar scan that abide to the alarm constraint to invoke the alarm (soft rock system tends to show more noise in coherence, hence five scan number is selected, while for hard rock system generally has a stable coherence measurement two scan number applied). Back-analysis of this alarm setting from each of the study cases will be presented afterward.

\subsection{Alarm back-analysis of study case 1}

The slope failure observed in this study case occurred at 8:46 pm on 24 November 2016. The alarm system is developed with coherence value at its main factor to trigger the alarm. The total amount of radar pixels showing progressive deformation behaviour that will eventually lead into the failure event is up to 120 pixels. The alarm then will be triggered if a certain amount of pixels, which, if determined from the percentage of the total pixels $(25,50$, and $75 \%)$, underwent deflation of coherence under the aforementioned coherence threshold $(0.999,0.995$, and 0.990$)$ on five successive occasion of radar scan. This setting yields nine specific times of alarm trigger time from which the elapse time of warning prior to failure can be inferred. The result of the alarm back-analysis for study case 1 shows a reasonable result of warning time across the different setting of coherence threshold. The nature of coherence shifting on this case allows quite flexible approach to detect the failure, partly due to the fact that this case is belong to the soft rock system. The back-analysis revealed that the recommended setting of alarm is using the 0.995 value as the coherence threshold over 25,50 , and $75 \%$ of the total pixels underwent progressive deformation behaviour. The warning time varied from $0.8,4.7$, and 8.3 hours. These period times is deemed ample to give mining team and other related party to conduct evacuation and other mitigation measure.

\subsection{Alarm back-analysis of study case 2}

Study case 2 is of soft rock designation as well hence it shows some degree of resemblance with study case 1 . The alarm setting on study case 1 could also be applied in study case 2 , albeit there is some notice. The start of the radar scan for this case is already half way of the progressive deformation behaviour. Thus, the coherence shifting did not represent a whole sequence from the beginning to end. Therefore, based on the alarm back-analysis, using the $25 \%$ of total pixels as the constraint yields almost the same result across three coherence thresholds. This result also suggests that the nature of the failure mechanism belongs to the creeping type, hence the coherence shifting tends to take a longer time before reaching the full percentage of coherence pixel deflation. The recommended setting based from the alarm back-analysis is applying 0.990 as the coherence threshold. It yield time span of 16, 40, and 63 hours prior to failure.

\subsection{Alarm back-analysis of study case 3}

Study case is an example of a hard rock system in which the coherence shifting tends to progress in a more rapid fashion than the case in a soft rock system. Hence, for this case, a difference alarm setting is introduced. The coherence threshold still clings to 0.999, 0.995, and 0.990, but other settings will be adjusted. The amount of pixels for the alarm to be triggered are 15,25 , and $50 \%$ of the total pixels from the area that has undergone progressive deformation behaviour, while the number of scans will be set into two. These are markedly affected by the typical coherence measurement on hard rock system areas which tend to be more acute and stable. The result from alarm back-analysis of this case suggests that the best possible coherence threshold to be applied is 0.999 with aforementioned additional setting. In turn, it will yield a warning time varied from $0.7,2.5$, and 2.7 hours. 


\section{Discussion}

The coherence application to detect impending failures as demonstrated above, both by intuitively observed coherence image shifting and applying the alarm system, to some extent will be overridden by its limitation. One must understand the circumstances in which the coherence shifting method presented in this paper becomes less reliable in detecting an impending failure. If the area of failure is not relatively large enough, this method will be less favourable. Automatically if this condition arises, the progression of the coherence shifting curve will be more steep and spiky, hence, the lapse time will be shorter and insufficient to provide warning. Other constraints for this method will also transpire if the area of failure is covered by vegetation. Vegetation will invoke an erratic signature of coherence measurement, therefore the coherence shifting curve will almost certainly be full of noise and will hardly represent a sequence of failure.

The same effect will also occur if the failure area is constantly disrupted by water seepage on the wall surface, a constant extreme atmospheric condition for a long time period, etc. Therefore, before applying this method, the radar engineer needs to attain full knowledge about the slope wall condition and other settings which will potentially hamper the accuracy of coherence value measurements by ground-based slope radar. Other things to emphasise is its dependency to progressive deformation behaviour identification prior to delineating the cluster as to the coherence shifting method could be established. In other words, detecting a failure using the coherence shifting method can be regarded as an aid to the conventional method to detect an imminent failure; that is, by observing progressive deformation behaviour. Moreover, on the case of ambiguity, as previously explained, progressive deformation behaviour observation will potentially give rise to incorrect interpretation. Coherence shifting method could give a better approach as to determine the exact time of failure event.

\section{Conclusion}

By applying the coherence shifting method along with the alarm system that has been explained, an impending failure could be detected at appropriate times. The timing of the warning notification from this method, which varied from 0.8 hours to more than 12 hours, provided ample time for any effort to ameliorate the severity of the failure hazard or other mitigate measure. This new method to detect impending failure, however, relies inherently with other conventional method that is detecting failure by observing its progressive deformation behaviour. The paramount thing to emphasise is the understanding of the person who analyse the radar data about the limitation of coherence which could hamper the detection itself. Notwithstanding that matter, detection with coherences shifting method could give enormous help to slope stability program applied on mining industry who deploy ground-based slope radar as its monitoring tool.

\section{Acknowledgement}

The author thanks GroundProbe and Orica for their support toward the submission of this paper.

\section{References}

Fukuzono, TA 1985, 'New method for predicting the failure time of a slope', in TA Fukuzono (ed.), Proceedings of the Fourth International Conference and Field Workshop on Landslides, Japan Landslide Society, Tokyo, pp. 145-150.

Goldstein, RM, Engelhardt, H, Kamb, B \& Frolich, RM 1993, 'Satellite radar interferometry for monitoring ice sheet motion: application to an Antarctic ice stream', Science, vol. 262, pp. 1525-1530.

Noon, D, Reeves, B, Stickley, G \& Longstaff, D 2001, 'Slope stability radar for monitoring mine walls', in C Nguyen (ed.), Proceedings of SPIE, vol. 4491, pp. 57-67.

Skolnik, MI 2001, Introduction to radar system, 3rd edn, Tata-Mcgraw-Hill, New York.

Zavodni, ZM \& Broadbent, CD 1980, 'Slope failure kinematics', Canadian Institute of Mining Metal Petroleum Bulletin, vol. 73 , issue 16 , pp. 69-74. 
New approach to detect imminent slope failure by utilising coherence attribute measurement on ground-based slope radar

FA Cahyo et al. 